Fluctuation and Noise Letters

Vol. X, No. X (2002) LXX-LXX

(C) World Scientific Publishing Company

\title{
ENTRAINMENT OF OPTICAL LOW-FREQUENCY FLUCTUATIONS IS ENHANCED BY COUPLING
}

\author{
J.M. BULDÚ, J. GARCÍA-OJALVO and M.C. TORRENT \\ Departament de Física i Enginyeria Nuclear, Universitat Politècnica de Catalunya, Colom 11, \\ 08222 Terrassa, Spain \\ RAÚL VICENTE, TONI PÉREZ and CLAUDIO R. MIRASSO \\ Departament de Física, Universitat de les Illes Balears, E-07071 Palma de Mallorca, Spain
}

\author{
Received (XX) \\ Revised (XX) \\ Accepted (XX)
}

\begin{abstract}
The control of the low-frequency fluctuations exhibited by two mutually coupled semiconductor lasers is studied experimentally and numerically. We observe that coupling enhances the response of the system to a weak periodic modulation of the injection current of one of the lasers, leading to a highly efficient entrainment of both laser outputs, in the form of synchronized and periodic power dropouts. We compare the quality of the entrainment with the one obtained in a single semiconductor laser with optical feedback, showing the beneficial role of coupling in this pursuit. The experimental observations are satisfactorily reproduced by numerical simulations of a set of coupled delay-differential rate equations.
\end{abstract}

Keywords: low-frequency fluctuations, periodic entrainment, current modulation, coupled semiconductor lasers, synchronization, bidirectional coupling

\section{Introduction}

Semiconductor lasers are devices prone to exhibit and be affected by different types of fluctuations. Their frequency noise is, for instance, fairly large in comparison with other kinds of lasers, which leads to a comparatively bigger emission linewidth. Furthermore, semiconductor lasers exhibit a rich dynamical behavior when affected by external influences. By way of example, under the influence of external reflections from its own light emission, they may undergo a dramatic increase in their linewidth $[1,2]$, a phenomenon called coherence collapse which has been associated to chaotic behavior. For weak feedback and operating conditions close to the lasing threshold a different regime appears, characterized by trains of fast pulses (at a time scale $\sim 100$ ps), modulated by sudden dropouts in power at much lower frequencies and ocurring at irregular times [3]. These low-frequency fluctuations (LFFs) have attracted much 
interest in recent years, with most studies devoted to understand their origin. Early theoretical work hinted at the deterministic origin of the fluctuations, relating them to the merging of attractor ruins of external-cavity modes and saddle-type points of the dynamics [4]. This indicates that noise, although unavoidably present in the system in the form of spontaneous emission, is not usually relevant for the LFF appearance (the situation is different when the system operates very close to the lasing threshold, where noise seems to play an important role [5]).

Besides the different works focused on explaining the origin of the LFFs, some efforts have been devoted to the control of this phenomenon. In this direction, the power dropouts exhibited by a semiconductor laser with feedback have been shown to be stabilized by a second external cavity $[8,9]$, and to become periodic when an external modulation is applied to the injection current $[10,11]$. Furthermore, we have recently shown [12] that coupling between two lasers greatly enhances the response of the system to the external harmonic modulation (similarly to what is found in general models of nonlinear media [13]), which leads to a very efficient entrainment: due to the fact that less pumping current is needed, the external modulation is practically absent in the output of the coupled system, so that the low-frequency dropouts are not distorted, but only entrained. In the present paper we present results obtained from a systematic analysis of this phenomenon, both experimental and numerical, discussing in particular the role of the modulation amplitude and the coupling strength between the two lasers. We also compare our results with the entrainment of the dropouts exhibited by a single semiconductor laser with optical feedback through modulation of the pumping current [10]. The outline of the paper is the following. Section 2 presents the experimental system and the dynamical regime being considered, and shows that the experimentally observed behavior can be described by a model of coupled delay-differential rate equations. Section 3 shows that efficient entrainment can be obtained by modulating the pump current of one of the lasers. Section 4 analyzes in a systematic way the effect of coupling, and Section 5 presents some conclusions.

\section{Synchronized low-frequency dropouts in coupled semiconductor lasers}

The experimental setup consists in two mutually coupled semiconductor lasers, as shown in Fig. 1. The two low-power $(\sim 5 \mathrm{~mW})$ ALGaInP semiconductor lasers emit at a wavelength of $650 \mathrm{~nm}$. Both lasers are temperature controlled with an accuracy of $\pm 0.01^{\circ} \mathrm{C}$ and pumped with a low-noise source of accuracy $\pm 0.1 \mathrm{~mA}$. The laser beams are collimated by two anti-reflection-coated laser objectives. A neutral density filter is placed between both lasers in order to control the coupling between them. In the detection branch two fast photodetectors send the signal to a $500 \mathrm{MHz}$ data acquisition card. The distance between the two lasers is $1.02 \mathrm{~m}$, which corresponds to a flying time of $\tau_{c}=3.4 \mathrm{~ns}$. The solitary laser thresholds are $I_{t h 1}=17.5 \mathrm{~mA}$ and $I_{t h 2}=17.3 \mathrm{~mA}$ at the temperatures of $T_{1}=18.15^{\circ} \mathrm{C}$ and $T_{2}=22.25^{\circ} \mathrm{C}$ respectively. Finally we are able to modulate the pumping current of the laser with a sinusoidal waveform introduced by an Agilent 33120 A function generator.

Previous investigations of this setup have revealed that coupling destabilizes the otherwise stable operation of the lasers, provided the detuning between their 


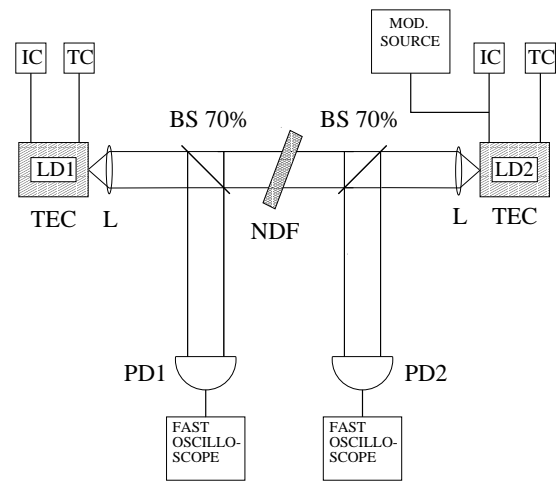

Fig 1. Experimental setup: LD, laser diode; BS, beam splitter; TEC, laser diode mount; PD, photodiode; IC, injection current source; TC, temperature controller; NDF, neutral density filter.

frequencies is small enough, and induces low-frequency fluctuations similar to those exhibited by a single laser with optical feedback [14]. Additionally, the resulting power dropout events are synchronized due to the coupling between the lasers. This situation is described in Fig. 2, which represents the experimental output of the two lasers (left) and the corresponding synchronization plot (right). In our case we adjust the temperature and pumping current to $I_{1}=17.8 \mathrm{~mA}, I_{2}=17.7 \mathrm{~mA}$, $T_{1}=18.15^{\circ} \mathrm{C}$ and $T_{2}=22.25^{\circ} \mathrm{C}$, which leads to a frequency detuning low enough to allow interaction between the two lasers.

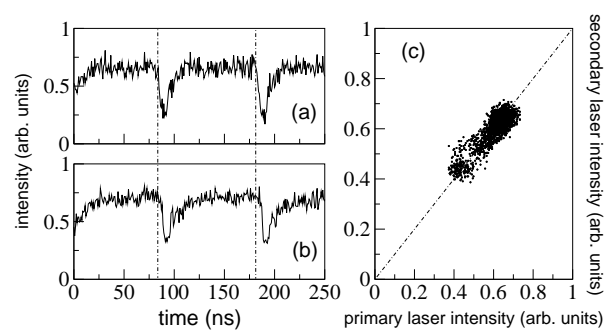

Fig 2. Experimental time traces corresponding to two mutually coupled lasers (a,b) and the corresponding synchronization plot (c). In (c) the output intensity of laser 2 has been advanced a time interval equal to the coupling time, i.e. the time the light takes to travel from one laser to the other.

The experimental results described above can be numerically reproduced by means of an extended rate-equation model for two mutually coupled semiconductor lasers. The model describes the evolution of the complex envelopes $E_{1,2}$ of the electric field and the carrier numbers $N_{1,2}$ [7], where the subindices 1 and 2 denote the different lasers:

$$
\begin{aligned}
& \frac{d E_{1,2}}{d t}=\frac{(1+i \alpha)}{2}\left[G_{1,2}-\gamma_{1,2}\right] E_{1,2} \pm i \Delta \omega E_{1,2}+\kappa e^{-i \Omega \tau_{c}} E_{2,1}\left(t-\tau_{c}\right) \\
& \frac{d N_{1,2}}{d t}=\frac{I_{1,2}}{e}-\gamma_{e 1, e 2} N_{1,2}-G_{1,2} P_{1,2}(t)
\end{aligned}
$$


Here $P_{1,2}(t)=\left|E_{1,2}(t)\right|^{2}$ is the photon number (optical intensity), and the laser parameters are the linewidth enhancement factor $\alpha$ (assumed equal for both lasers), the cavity loss coefficients $\gamma_{1,2}$ and the carrier decay rates $\gamma_{e 1, e 2}$. The last term in Eq. (1) corresponds to the coupling between the lasers, where $\kappa$ is the coupling coefficient, $\tau_{c}$ is the time that takes the light to go from one laser to the other and $\Omega=\left(\omega_{1}+\omega_{2}\right) / 2$ is the reference frequency, with $\omega_{1}$ and $\omega_{2}$ being the corresponding free-running optical frequencies of each laser. The gain coefficients $G_{1,2}$ are calculated by the following expression:

$$
G_{1,2}(t)=\frac{\left[g\left(N_{1,2}-N_{0}\right)\right]}{\left[1+s P_{1,2}(t)\right]},
$$

where $g$ is the differential gain coefficient, $N_{0}$ is the carrier number at transparency and $s$ is the gain saturation factor. The values used in the simulations are the following: $\alpha=3.5, \kappa=20 \mathrm{~ns}^{-1}, \tau_{c}=3.4 \mathrm{~ns}, g=1.2 \times 10^{-8} \mathrm{ps}^{-1}, s=5 \times 10^{-7}$ and $N_{0}=1.25 \times 10^{8}$. We have chosen different values for the cavity losses and the cavity decay rates in order to match the threshold currents with the values observed in the experiments $\left(I_{t h 1}=17.3 \mathrm{~mA}\right.$ and $\left.I_{t h 2}=17.5 \mathrm{~mA}\right)$. In this case $\gamma_{1}=0.687 \mathrm{ps}^{-1}, \gamma_{2}=0.496 \mathrm{ps}^{-1}, \gamma_{e 1}=0.601 \mathrm{~ns}^{-1}$ and $\gamma_{e 2}=0.651 \mathrm{~ns}^{-1}$. We also introduce a slight detuning $\Delta \omega=\left(\omega_{1}-\omega_{2}\right) / 2=2 \mathrm{GHz}$ to mimic the experimental conditions of Fig. 2, where a nonzero detuning was introduced (see below). The external modulated signal is introduced in the system through the pumping current of laser 1 as $I_{1}=I_{b 1}+A_{m} \sin \left(2 \pi t / T_{m}\right)$, where $I_{b 1}=I_{t h 1}$ is the constant part of the pumping current and $A_{m}=0.3$ and $T_{m}=100$ ns are the amplitude and period of the modulated signal. Laser 2 is pumped with a constant current $I_{2}=I_{t h 2}$.

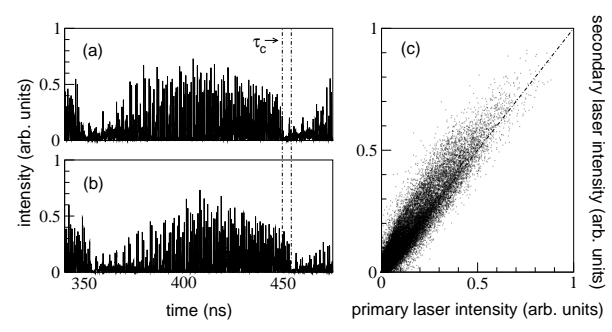

Fig 3. Numerical results of model (1)-(2) corresponding to two mutually coupled lasers. Note that the intensity has not been filtered in order to observe the fast intensity pulses exhibited by the lasers. The parameters of the model are given in the text. In (c) the output intensity of laser 2 has been advanced a time interval $\tau_{c}$.

Figure 3 shows the results obtained from a numerical simulation of model (1)-(2) with the parameters given above. The time traces of the light emitted by both lasers (left) is shown together with a corresponding synchronization plot (right). Note that the time series have not been filtered, in order to expose the fast pulsing involved in the low-frequency fluctuation regime. In this way, the figure shows that the dynamical behavior considered here involve two vastly different time scales: a slow one corresponding to the low-frequency fluctuations, and a fast one related to the fast pulses between the dropouts. This behavior would be observed experimentally if a detector with large enough bandwidth were used, as has already been shown in the 
case of a single semiconductor laser with optical feedback [2]. We can thus conclude that the numerical results obtained from the delay-differential rate-equation model satisfactorily reproduce the experimental observations. An important feature shown in Fig. 3 is that in spite of the good degree of synchronization, the dropouts in the two lasers do not occur simultaneously. In fact, there is a delay between them equal to the coupling time $\tau_{c}$. The situation is also present in the experimental results, as can also be seen in Fig. 2 by examining the vertical dashed lines. This has been taken into account in the synchronization plots of the two figures, where the output intensity of the second laser has been advanced $\tau_{c}$. The good quality of the two plots indicates that this delay is systematic. The experimental results indicate that the intensity of the laser with higher frequency will always drop before that of the laser with lower frequency [14]. Hence, a leader-laggard dynamics is established in this system: due to the detuning, we can make one laser the leader (laggard) in the dynamics by decreasing (increasing) its wavelength.

\section{Entraining low-frequency dropouts with coupling and modulation}

We now aim to control the power dropouts described in the previous Section by inducing them to occur periodically. Following the ideas of previous works in single semiconductor lasers with optical feedback [10], we add a $10-\mathrm{MHz}$ harmonic modulation to the pumping current of one of the lasers. In the results that follow, the external modulation will be apply to the leader laser, but similar results are found when we modulate the laggard laser [12]. The experimental response of the two lasers to the harmonic driving is shown in Fig. 4 for increasing amplitude of the modulation. For small amplitudes the situation is similar to the free-running case, with power dropouts synchronized (with a delay $\tau_{c}$ ) and occurring at irregular times (upper plot). As the modulation amplitude increases, the time intervals between dropouts occur more regularly (middle plot), until for large enough amplitudes the dropouts are completely entrained to the external signal, with one dropout occurring at every period of the modulation (lower plot). In the lower plot of Fig. 4, a

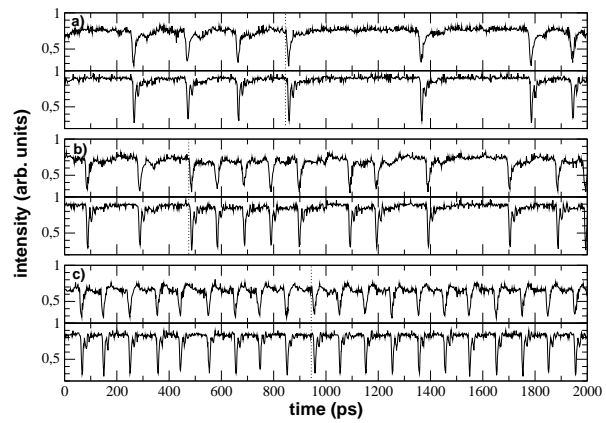

Fig 4. Experimental time series of the two lasers for increasing values of the external modulation amplitude: from top to bottom, $0.125 \mathrm{~mA}, 0.250 \mathrm{~mA}$ and $0.500 \mathrm{~mA}$. The upper time trace in each plot corresponds to the modulated laser. Note the filtering action of the laggard laser (lower time traces), where modulation is smoothed.

slight modulation in the output intensity of the leader (directly modulated) laser can be found, but this modulation is absent in the laggard. This is due to the fact 
that the laggard laser is synchronized with the intrinsic dynamics of the leader and not with the modulation (a standard effect in chaos synchronization [16]).

As shown in Fig. 4, the output modulation of the directly driven laser is very small. This is related to the fact that the harmonic modulation needed to produce the entrainment is very small (less than $3 \%$ of the baseline current). It is worth at this time to compare this effect with the one observed in the case of a single laser with optical feedback. In that case power dropouts are also produced, and have been shown to be entrained by direct modulation of the pump current of the laser [10]. Figure 5 compares this entrainment (a) with the one obtained in two mutually coupled semiconductor lasers (b). In this particular example, the modulation amplitude is $17.1 \%$ of the threshold current in the case of the single laser with optical feedback (a), and $2.8 \%$ in the case of the two mutually coupled lasers (b). The fact the required modulation amplitude is much larger in the former than in the latter case (no entrainment can be obtained in (a) for weaker modulation) leads to a substantial perturbation of the laser output in (a), whereas in (b) the entrainment does not affect basically the dynamics between dropouts. This result can be interpreted as an enhancement of the response of the nonlinear system to a harmonic driving due to coupling between the elements [13]. We note that Fig. 5(b) shows the time trace of the laggard laser output, which is not directly modulated (although the behavior of the leader laser is very similar, as was shown in Fig. 4).

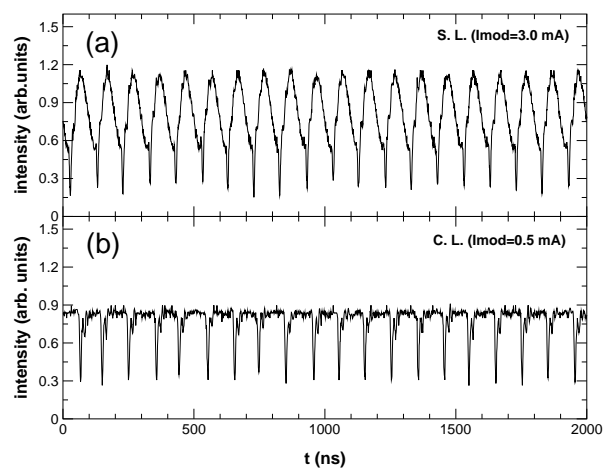

Fig 5. Intensity time trace of (a) a single laser with optical feedback and (b) of the laggard laser in a mutually coupled configuration. Both systems are in the entrained LFF regime.

\section{Effect of coupling strength}

In order to quantify the amount of coupling needed to observe the entrainment, we have studied the response of the system to a varying coupling strength by placing a neutral density filter between the two lasers. Starting from the system perfectly entrained at the maximum coupling (i.e. when the amount of light that is injected in one laser from the other is maximum given our experimental conditions - beamsplitters, collimators, etc), we have steadily reduced the coupling between the lasers. The experimental results are presented in Fig. 6, where it can be seen that for intermediate values of coupling the entrainment persists [plot (b)], and only when the coupling strength is reduced more than $50 \%$ of its maximum value, the quality 
of the entrainment is noticeably degraded [plot (c)]. The results are also given in terms of the probability distribution function of the time intervals between consecutive dropouts. The irregular shape of this function in Fig. 6(c) indicates loss of entrainment. This behavior can be reproduced by a numerical integration of model (1)-(2), as shown in Fig. 7. In these numerical time series, the output intensity has been filtered with a $500 \mathrm{MHz}$ Butterworth filter with the aim of reproducing the effect of the experimental photodetector.

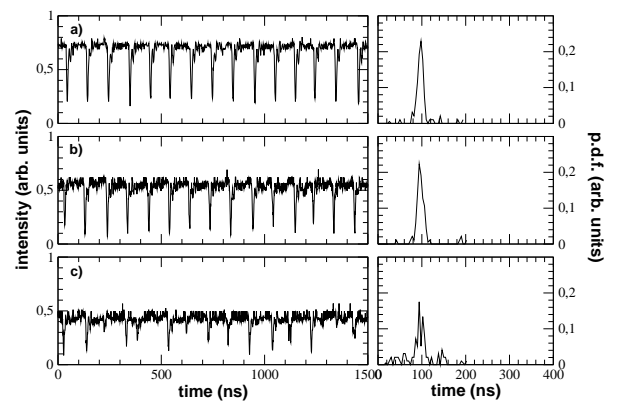

Fig 6. Experimental time series of the laggard laser when the coupling between leader and laggard is decreased. In relation with the maximum coupling: (a) $100 \%$, (b) $83.9 \%$ and (c) $45.8 \%$. The right plots show the corresponding probability distribution functions of the intervals between dropouts.

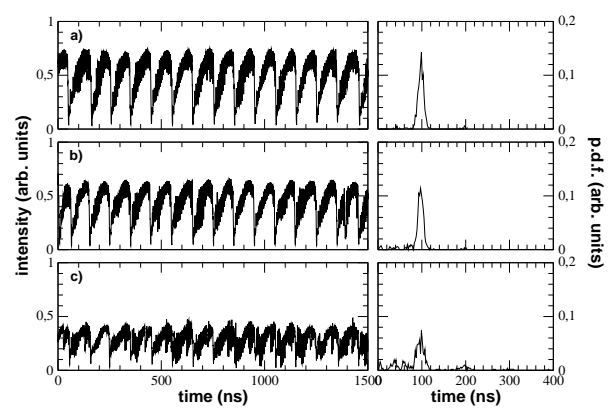

Fig 7. Numerical results corresponding to the experimental observations presented in Fig. 6.

\section{Conclusions}

In conclusion, we have analyzed the entrainment of the power dropouts exhibited by two mutually coupled semiconductor lasers in the presence of harmonic modulation of one of the pumping currents. We have focused our study in the effects of the coupling, concluding that coupling enhances the response of the system to an harmonic external signal. We observe advantages in using a coupled system in front of a single one, from the point of view of the quality and efficiency of the entrainment. Numerical simulations obtained from a model of coupled rate equations with delay coupling terms reproduce satisfactorily the observed experimental results, from the point of view of the synchronization and the coupling efficiency. 
Buldú, García-Ojalvo, Torrent, Vicente, Pérez and Mirasso

\section{Acknowledgments}

We acknowledge financial support from the Ministerio de Ciencia y Tecnologia (Spain) and FEDER, under projects BFM2000-1108, BFM2001-0341, BFM20012159, and BFM2002-04369, from the EC project OCCULT IST-2000-29683, and from the Generalitat de Catalunya (project 2001SGR00223).

\section{References}

[1] D. Lenstra, B. Verbeek and A.J. de Boef, IEEE J. Quantum Electron. 21, 764 (1985).

[2] I. Fischer, G.H.M. van Tartwijk, A.M. Levine, W. Elsäßer, E. Göbel and D. Lenstra, Phys. Rev. Lett. 76, 220 (1996).

[3] Ch. Risch and C. Voumard J. Appl. Phys. 48, 2083 (1977).

[4] T. Sano, Phys. Rev. A 50, 2719 (1994).

[5] R. Roy, private communication.

[6] R. Lang and K. Kobayashi, IEEE J. Quantum Electron. 16, 347 (1980).

[7] T. Heil, I. Fischer, W. Elsäßer, J. Mulet and C.R. Mirasso, Opt. Lett. 24, 1275 (1999).

[8] F. Rogister, P. Mégret, O. Deparis, M. Blondel and T. Erneux, Opt. Lett 24, 1218 (1999).

[9] F. Rogister, D.W. Sukow, A. Gavrielides, P. Mégret, O. Deparis, M. Blondel, Opt. Lett 24, 1218 (1999).

[10] D.W. Sukow and D.J. Gauthier, IEEE J. Quantum Electron. 36, 175 (2000).

[11] J.M. Buldú, J. García-Ojalvo, C.R. Mirasso, M.C. Torrent, and J.M. Sancho, Phys. Rev. E 64, 051109 (2001); J.M. Buldú, J. García-Ojalvo, C.R. Mirasso, and M.C. Torrent, Phys. Rev. E 66, 021106 (2002).

[12] J.M. Buldú, R. Vicente, T. Pérez, C.R. Mirasso, M.C. Torrent, and J. García-Ojalvo, Appl. Phys. Lett., to appear (2002).

[13] J.F. Lindner, B.K. Meadows, W.L. Ditto, M.E. Inchiosa and A.R. Bulsara, Phys. Rev. Lett. 75, 3 (1995).

[14] T. Heil, I. Fischer, W. Elsäßer, J. Mulet, and C.R. Mirasso, Phys. Rev. Lett. 86, 795 (2001).

[15] J. Mulet, C. Masoller, and C.R. Mirasso, Phys. Rev. A 65, 063815 (2002).

[16] C.R. Mirasso, P.Colet and P. Garcia-Hernandez, IEEE Phot. Tech. Lett. 8, 299 (1996). 